\title{
Carbapenemases on the move: it's good to be on ICEs
}

\author{
João Botelho ${ }^{1 *}$ (D) Adam P. Roberts ${ }^{2,3}$, Ricardo León-Sampedro ${ }^{4,5}$, Filipa Grosso ${ }^{1}$ and Luísa Peixe ${ }^{1^{*}}$
}

\begin{abstract}
Background: The evolution and spread of antibiotic resistance is often mediated by mobile genetic elements. Integrative and conjugative elements (ICEs) are the most abundant conjugative elements among prokaryotes. However, the contribution of ICEs to horizontal gene transfer of antibiotic resistance has been largely unexplored.

Results: Here we report that ICEs belonging to mating-pair formation (MPF) classes $G$ and T are highly prevalent among the opportunistic pathogen Pseudomonas aeruginosa, contributing to the spread of carbapenemaseencoding genes (CEGs). Most CEGs of the $\mathrm{MPF}_{\mathrm{G}}$ class were encoded within class I integrons, which co-harbour genes conferring resistance to other antibiotics. The majority of the integrons were located within Tn3-like and composite transposons. Conserved attachment site could be predicted for the MPF G Class ICEs. MPF Class ICEs carried the CEGs within composite transposons which were not associated with integrons.

Conclusions: The data presented here provides a global snapshot of the different CEG-harbouring ICEs and sheds light on the underappreciated contribution of these elements to the evolution and dissemination of antibiotic resistance on $P$. aeruginosa.
\end{abstract}

Keywords: Integrative and conjugative elements, Carbapenemases, Pseudomonas spp., Antibiotic resistance

\section{Background}

Among the non-fermenting Gram-negative bacteria, the Pseudomonas genus is the one with the highest number of species $[1,2]$. Pseudomonas aeruginosa, an opportunistic human pathogen associated with an ever-widening array of life-threatening acute and chronic infections, is the most clinically relevant species within this genus [3-5]. $P$. aeruginosa is one of the CDC "ESKAPE" pathogens Enterococcus faecium, Staphylococcus aureus, Klebsiella pneumoniae, Acinetobacter baumannii, P. aeruginosa and Enterobacter species -, emphasizing its impact on hospital infections and the ability of this microorganism to "escape" the activity of antibacterial drugs [6]. P. aeruginosa can develop resistance to a wide range of antibiotics due to a combination of intrinsic, adaptive, and acquired resistance mechanisms, such as the reduction of its outer membrane permeability, over-expression of constitutive or inducible efflux pumps, overproduction of AmpC

\footnotetext{
* Correspondence: joaobotelho9@hotmail.com; Ipeixe@ff.up.pt

${ }^{1}$ UCIBIO/REQUIMTE, Laboratório de Microbiologia, Faculdade de Farmácia da Universidade do Porto, Rua Jorge Viterbo Ferreira n 228, 4050-313 Porto, Portugal

Full list of author information is available at the end of the article
}

cephalosporinase, and the acquisition of antibiotic resistance genes (ARGs) through horizontal gene transfer (HGT) $[4,7,8]$. P. aeruginosa has a non-clonal population structure, punctuated by specific sequence types (STs) that are globally disseminated and frequently linked to the dissemination of ARGs [4, 9]. These STs have been designated as high-risk clones, of which major examples are ST111, ST175, ST235 and ST244.

Due to its high importance for human medicine, carbapenems are considered by the World Health Organization as Critically-Important Antimicrobials that should be reserved for the treatment of human infections caused by MDR Gram-negative bacteria [10], such as P. aeruginosa. Carbapenem-resistant $P$. aeruginosa is in the "critical" category of the World Health Organization's priority list of bacterial pathogens for which research and development of new antibiotics is urgently required [11]. Besides $P$. aeruginosa, carbapenem resistance has been reported in other Pseudomonas spp. and is often mediated by the acquisition of carbapenemase-encoding genes (CEGs) [12-14]. Carbapenemases are able to hydrolyse carbapenems and confer resistance to virtually all $ß$-lactam antibiotics [15]. In the Pseudomonas genus, CEGs are mostly 
present on class I integrons within the chromosome [4]. Class I integrons are genetic elements that carry ARGs and an integrase gene, which controls integration and excision of genes [16-18]. Mobile genetic elements (MGEs) such as transposons, plasmids and integrative and conjugative elements (ICEs), are responsible for the spread of ARGs [19-23].

Usually, the genes acquired by HGT are integrated in common hotspots in the host's chromosome, comprising a cluster of genes designated by genomic islands (GIs) $[19,24,25]$. This broad definition also encompass other MGEs, such as ICEs and prophages. ICEs are self-transmissible mosaic and modular MGEs that combine features of transposons and phages (ICEs can integrate into and excise from the chromosome), and plasmids (ICEs can also exist as circular extrachromosomal elements, replicate autonomously and be transferred by conjugation) [21, 24, 26-29]. Integrative and mobilizable elements encode their own integration and excision systems, but take advantage of the conjugation machinery of co-resident conjugative elements to be successfully transferred [30]. ICEs usually replicate as part of the host genome and are vertically inherited, remaining quiescent, and with most mobility genes repressed [31, 32]. These elements also encode recombinases related to those in phages and other transposable elements. Conjugation involves three mandatory components: a relaxase, a type-IV secretion system (T4SS) and a type-IV coupling protein (T4CP) [33, 34]. Four mating-pair formation (MPF) classes cover the T4SS among Proteobacteria: $\mathrm{MPF}_{\mathrm{T}}, \mathrm{MPF}_{\mathrm{G}}, \mathrm{MPF}_{\mathrm{F}}$ and $\mathrm{MPF}_{\mathrm{I}}$ [35]. The first is widely disseminated among conjugative plasmids and ICEs, while $\mathrm{MPF}_{\mathrm{F}}$ is more prevalent in plasmids of $\gamma$-Proteobacteria and $\mathrm{MPF}_{\mathrm{G}}$ is found essentially on ICEs. MPF ${ }_{I}$ is rarely identified. Guglielmini et al. constructed a phylogenetic tree of VirB4, a highly conserved ATPase from the T4SS apparatus of different conjugative plasmids and ICEs, and formulated the hypothesis of interchangeable conjugation modules along their evolutionary history [36]. A close interplay between these elements in the ancient clades of the phylogenetic tree was observed, suggesting that plasmids may behave like ICEs and vice-versa, reinforcing the common assumption that the line separating ICEs and conjugative plasmids is blurring $[27,37]$. These authors also searched more than 1000 genomes and found that ICEs are present in most bacterial clades and are more prevalent than conjugative plasmids [36]. It was also observed that the larger the genome, the higher the likelihood to harbour a conjugative element at a given moment, which supports the common assumption that bacteria with large genomes are more prone to acquire genes by HGT [38-40].

Delimiting ICEs in genomic data remains particularly challenging [25]. Some signatures features are frequently observed, such as a sporadic distribution, sequence composition bias, insertion next to or within a tRNA gene, bordering attachment $(\mathrm{att})$ sites and over-representation of mobility genes of the T4SS. However, some ICEs present atypical features and may not be detected by these approaches $[25,38]$. In $P$. aeruginosa, most ICEs fall into three large families: the ICEclc, pKLC102 and Tn4371. The PAGI2(C), PAGI3(SG), PAGI-13, PAGI-15 and PAGI-16 were previously described as members of the ICEclc family, while the PAPI-1, PAPI-2, PAGI-4 and PAGI-5 were linked to the pKLC102 family [19]. The $\mathrm{ICE}_{\mathrm{Tn} 4371}$ family also represents a large group of ICEs with a common backbone and which are widely distributed, such as in P. aeruginosa UCBPP-PA14, PA7 and PACS171b strains [21]. These ICEs have been frequently implicated in virulence $[41,42]$.

Previous reports characterized the complete nucleotide sequence of extra-chromosomal genetic elements housing different CEGs in pseudomonads [20, 43-46]; however, the association of CEGs with chromosome-located MGEs has rarely been investigated [47-49]. Taking into consideration that i) in pseudomonads, CEGs are frequently located within the chromosome, ii) ICEs are the most abundant conjugative elements in prokaryotes and iii) ICEs are more frequently identified in large bacterial genomes, such as in pseudomonads, we hypothesize that ICEs may play a key role in the horizontal spread of CEGs. To investigate this hypothesis, we developed an in silico approach to explore the association between ICEs and CEGs in pseudomonads.

\section{Results}

\section{A plethora of carbapenemase-encoding genes was} identified in a subset of Pseudomonas species

From the total Pseudomonas genomes analysed $(n=4565)$, 313 CEGs were identified in 297 genomes (Fig. 1 and Additional file 1: Table S1). As expected, bla $a_{\mathrm{VIM}-2}$ represents the majority of the CEGs found among Pseudomonas spp., being detected mainly in $P$. aeruginosa, followed by $P$. plecoglocissida, $P$. guariconensis, $P$. putida, $P$. stutzeri and 16 genomes corresponding to unidentified species (Additional file 1: Table S1). Curiously, some strains presented two CEGs, either presenting a duplication of the


NCGM 1984 Japanese isolates, or harbouring different CEGs, such as bla $a_{\mathrm{IMP}-1}$ and $b l a_{\mathrm{DIM}-1}$ in isolates 97,130 and 142 recovered in Ghana (Additional file 1: Table S1, highlighted in red). A wide variety of STs was also observed, including the high-risk clones ST111, ST175 and ST244.

\section{Detection of ICE encoding carbapenemases in Pseudomonas spp}

65.5\% (205/313, Additional file 1: Table S1) of the CEG hits are located within small contigs, with a sequence 


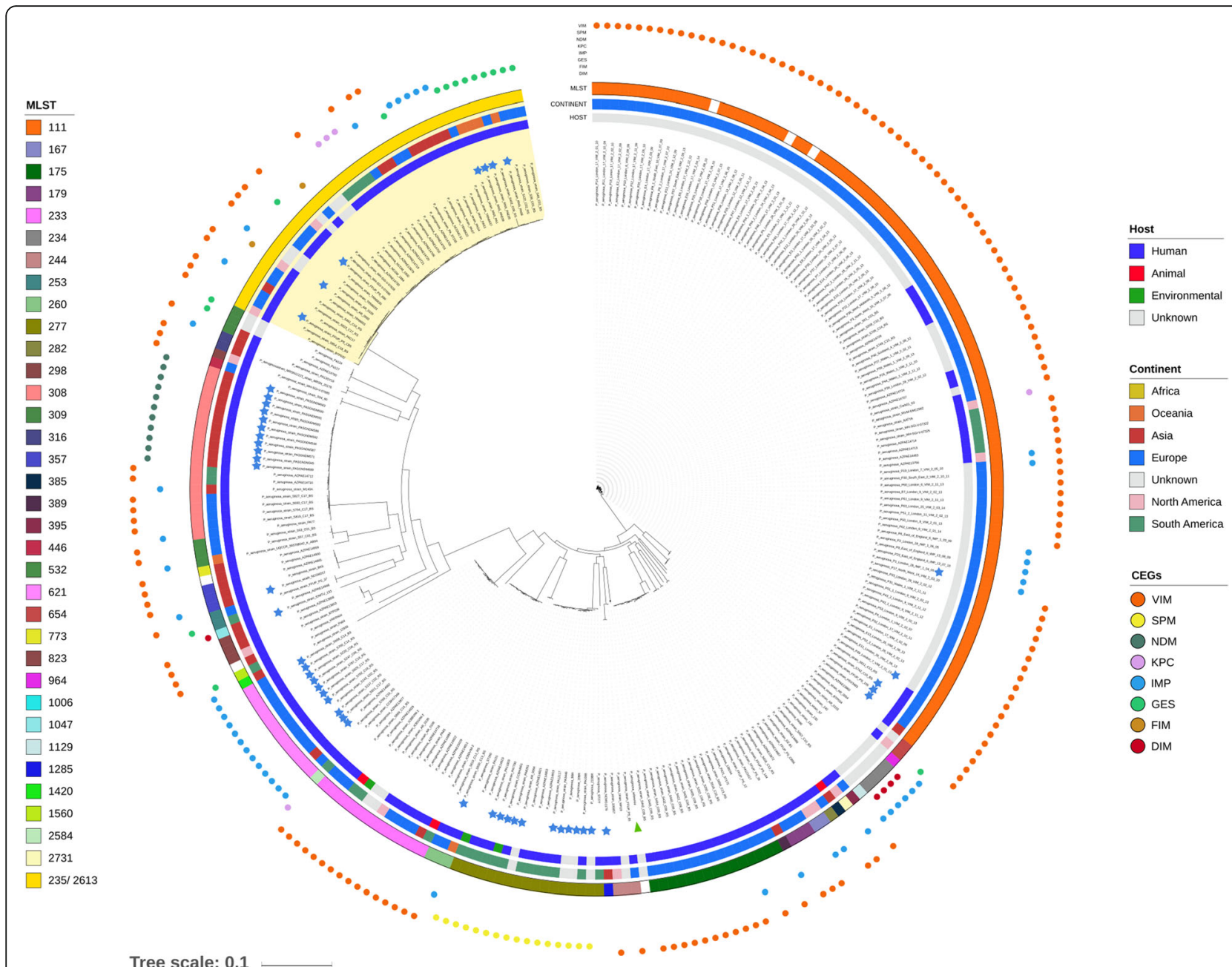

Fig. 1 Whole-genome phylogeny of the CEG-carrying P. aeruginosa isolates. The maximum-likelihood phylogenetic tree was constructed using 146,106 single nucleotide polymorphisms (SNPs) spanning the whole genome and using the P. aeruginosa PAO1 genome (highlighted by a green triangle) as a reference. Multilocus sequence typing (MLST), continent and host data are reported on the outer-most, middle and innermost circles, respectively. The strains belonging to a double ST profile (ST235/ST2613) are shaded yellow. Blue stars point out $P$. aeruginosa strains for which a CEG-harbouring ICE was predicted. The P. aeruginosa AR_0356 genome (accession number CP027169.1) was removed from the tree since it corresponds to a strain of which host and origin are unknown. The phylogenetic distance from the tree root to this genome is 1 (calculated with the tree scale). The Newick format file for the original tree is included in the Additional file 3

smaller than $20 \mathrm{~kb}$ in length. The presence of repeated regions, such as those encoding for transposases, tends to split the genome when second-generation sequencing approaches are used. Based on information retrieved from NCBI (accessed on the 24th of May, 2018), the total number of bacterial genomes sequenced at the chromosome/complete genome level is 12,077 , while the number of genomes sequenced at the scaffold/contig is much larger $(127,231)$. With this sequencing limitation, we were still able to identify 49 ICEs associated with CEGs ( $n=20$ with complete sequence) among all pseudomonads genomes (Table 1, Additional file 1: Table S1 and Fig. 1). When we attributed an ICE location to a CEG located on a small contig, this assumption was based on previously published data, as pointed out on
Table 1. Besides the aforementioned ICEs, we also identified a putative MGE within Pseudomonas sp. NBRC 111143 strain (Additional file 1: Table S1). The T4CP-encoding gene was absent from this bla $_{\mathrm{IMP}-10^{-}}$carrying element, which could be due to contig fragmentation or gene absence. In case the gene is actually missing, this element could still be mobilized by the conjugation machinery of an ICE or conjugative plasmid(s) present in the host, and should be classified as an integrative and mobilizable element.

The ICEs identified here were all integrated within $P$. aeruginosa genomes (with the exception of the one element identified in Pseudomonas sp. PONIH3 genome) and AT-rich when compared to their host's chromosome; the mean GC value for this species is 


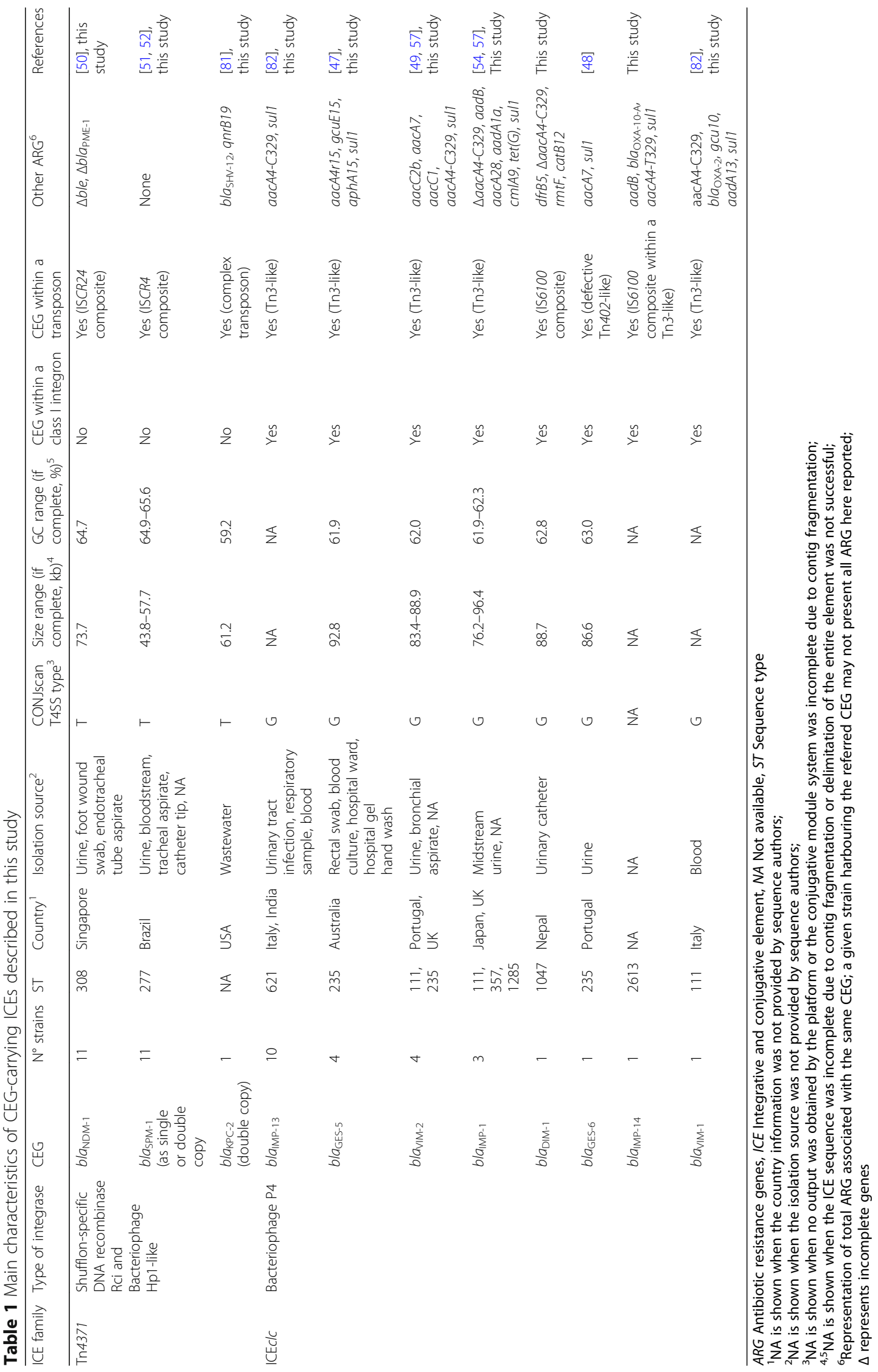


66.2\% according to EZBioCloud (https://www.ezbiocloud. net/taxon?tn=Pseudomonas\%20aeruginosa) (Table 1).

All ICEs identified here possessed only one tyrosine integrase (Fig. 2). ICEs belonging to the ICEclc family $\left(\mathrm{MPF}_{\mathrm{G}}\right.$ class) carried an integrase belonging to the bacteriophage P4-like family, while ICEs belonging to the $\mathrm{ICE}_{\mathrm{Tn} 4371}$ family $\left(\mathrm{MPF}_{\mathrm{T}}\right.$ class) carried an integrase belonging to shufflon-specific DNA recombinase Rci and Bacteriophage Hp1-like family (Table 1). Rci and Hp1-like were only distantly related (13\% amino acid identity) to P4-like integrases. Orthologous assignment of these integrases revealed that the former and the later integrases identified were present in more than 100 and 400 proteobacteria species, respectively. While P4-like integrases were more prevalent on $\gamma$-proteobacteria, half of the strains carrying Rci and Hp1-like integrases belong to the $\alpha$-proteobacteria.

We observed that $\mathrm{MPF}_{\mathrm{G}}$ class ICEs tend to integrate into a single copy of tRNA ${ }^{\text {Gly }}$ or a cluster of two tRNAGlu and one tRNA ${ }^{\text {Gly }}$ genes, which is in agreement with previous findings [25, 38]. A conserved 8-bp att site (5'-CCGCTCCA) flanked all complete ICEs of the $\mathrm{MPF}_{\mathrm{G}}$ class identified here (Table 1). Notably, most ICEs of this class were adjacent to phages (either at the $5^{\prime}$ - or the $3{ }^{\prime}$-end) targeting the same att site as the neighbour ICE. No att site could be identified for the integration of $\mathrm{MPF}_{\mathrm{T}}$ class ICEs. A gene encoding for a catechol 1,2-dioxygenase and a gene encoding for a protein with no described conserved domain were found flanking the $b l a_{\mathrm{SPM}-1}$-harbouring ICEs. Regarding the elements carrying $b l a_{\mathrm{NDM}-1}$, a gene encoding for a different protein also with no conserved domain identified and a gene encoding for the type III secretion system adenylate cyclase effector ExoY were separated upon insertion of these ICEs. Integration next to hypothetical proteins or tRNA genes was commonly observed.

Carbapenemases are frequently encoded within transposons CEGs were associated with class I integrons frequently co-harbouring aminoglycoside resistance genes when associated with MPF class G ICEs (Table 1). Class I integrons were often associated with a wide array of transposons, such as the Tn3 superfamily transposons and the IS6100 composite elements (Table 1). MPF class ICEs were targeted by more complex elements, such as the composite transposons carrying bla $a_{\mathrm{SPM}-1}$ and bla NDM-1 $_{\text {(Table } 1) .}$

The bla $a_{\text {NDM-1 }}$ gene was identified in an isolate from Singapore in $\mathrm{ICE}_{\mathrm{Tn} 4371} 6385$ and associated with ST308, as recently reported [50]. The $b l a_{\mathrm{NDM}-1}$ was flanked by two ISCR24-like transposases. bla $a_{\mathrm{SPM}-1}$ was linked to $\mathrm{ICE}_{\mathrm{Tn} 4371} 6061$, a recently described ICE [51]. Again, the CEG was located within an ISCR4-like composite transposon. ISCR elements are atypical elements of the IS91 family which represent a well-recognized system of gene capture and mobilization by a rolling-circle transposition process $[21,52]$.

Besides previously described bla $a_{\mathrm{NDM}-1}$ and $b l a_{\mathrm{SPM}-1}$ harbouring ICEs, we characterize here new ICEs of $\mathrm{MPF}_{\mathrm{G}}$ and $\mathrm{MPF}_{\mathrm{T}}$ classes (Table 1 and Fig. 3). The

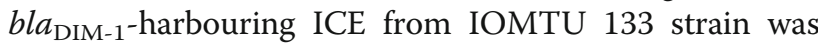
integrated into the 3 '-end of a tRNA ${ }^{\text {Gly }}$ gene (IOMTU133_RS11660) and next to a gene encoding for the $R$ body protein RebB (IOMTU133_RS12085). bla DIM-1 was first described as a two gene cassette (found together with $a a d B$; encoding resistance to aminoglycosides) located within a class I integron associated with a 70-kb Pseudomonas stutzeri plasmid recovered in the Netherlands [13]. However, the integron carrying blaDIM-1 in strain IOMTU 133 was unrelated to the one from the $P$. stutzeri plasmid, harbouring $b l a_{\mathrm{DIM}-1}$ as a single gene cassette plus genes encoding for aminoglycoside (aacA4-C329 and rmtf), trimethoprim (dfrB5) and chloramphenicol (catB12) resistance (Fig. 3a). Direct repeats (DRs) were found flanking the entire IS6100 composite transposon (5'-TTCGAGTC), indicating the transposition of this element into the ICE. Besides being identified as a composite transposon, IS6100 was frequently observed as a single copy at the 3'end of the class I integron (Fig. $3 \mathrm{~b}$ and c), suggesting that these elements were derived from the In4 lineage [53]. The bla IMP-1 $_{1}$ from the NCGM257 strain identified in Japan belonged to a different ST (ST357) than the frequently identified ST235 associated with the spread of this CEG in this country [54]. The CEG was also shown to be associated with a novel class I integron, co-harbouring $a a d B$, cmlA9 and tet(G) genes encoding resistance to aminoglycosides, chloramphenicol and tetracyclines, respectively (Fig. 3b). This integron was inserted (DRs 5'- GAGTC) within a mercury resistance transposon. This genetic organization was frequently recovered among other ICE-harbouring strains, such as the ones associated with $b l a_{\mathrm{GES}-5}, b l a_{\mathrm{IMP}-13}$ and $b l a_{\mathrm{IMP}-14}$ (Table 1). The entire ICE was integrated in the chromosome of NCGM257 strain into the 3 ' -end of a tRNA ${ }^{\text {Gly }}$ gene (PA257_RS24790) and next to a Pseudomonas phage Pf1-like element. The new ICE identified on the P1_London_28_IMP_1_04_05 strain presented $b l a_{\mathrm{IMP}-1}$ in a different In4-like integron than that observed for the NCGM257 strain, even though both elements were associated with a Tn3-like transposon (Fig. 3c). Unlike most ICEs of the $\mathrm{MPF}_{\mathrm{G}}$ class, its integration occurred between a gene encoding for a LysR family transcriptional regulator (AFJ02_RS19410) and a gene encoding for a hypothetical protein (AFJ02_RS19770). Regarding the bla KPC-2-harbouring Pseudomonas sp. PONHI3 strain, an average nucleotide identity based on BLAST (ANIb) analysis revealed that this strain belongs to the Pseudomonas 


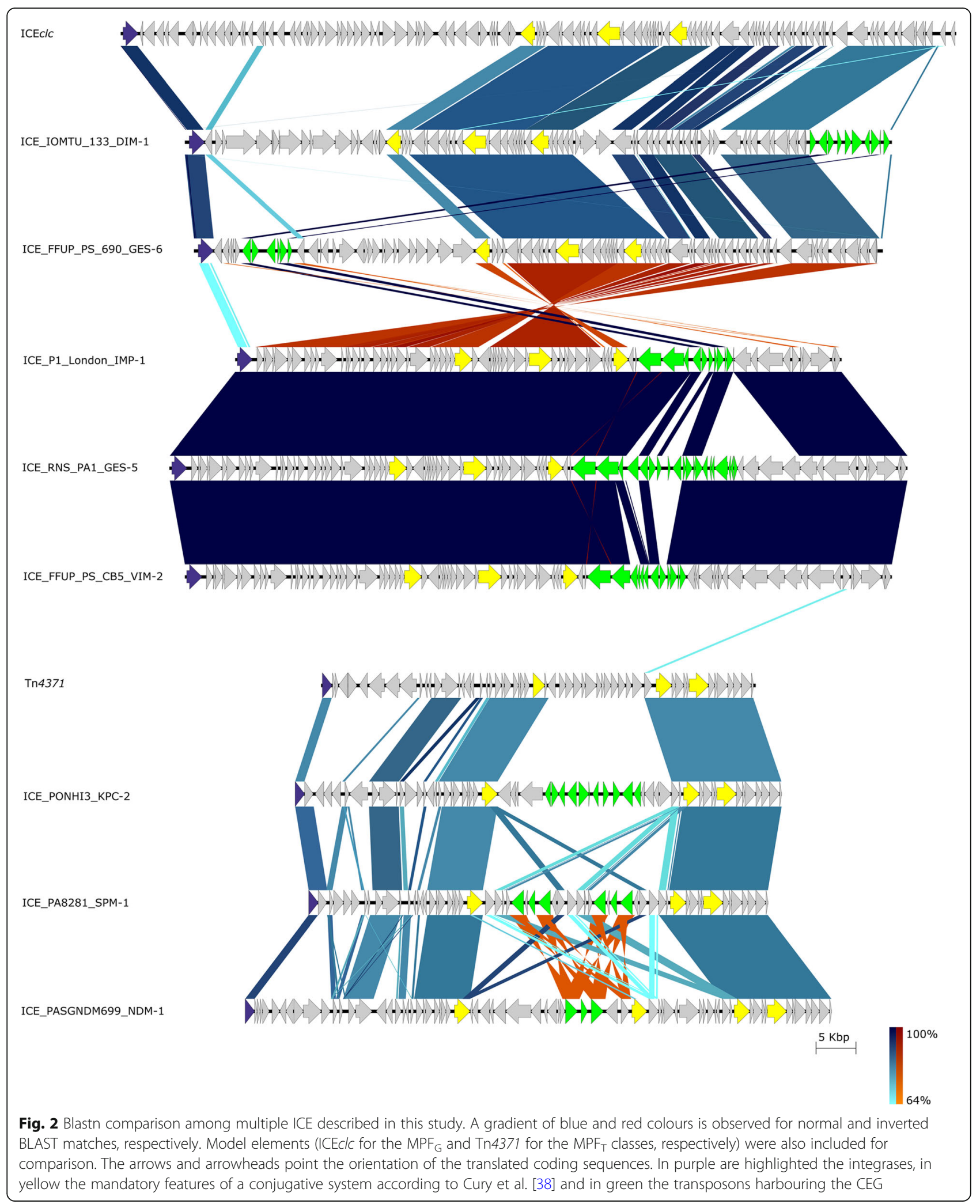




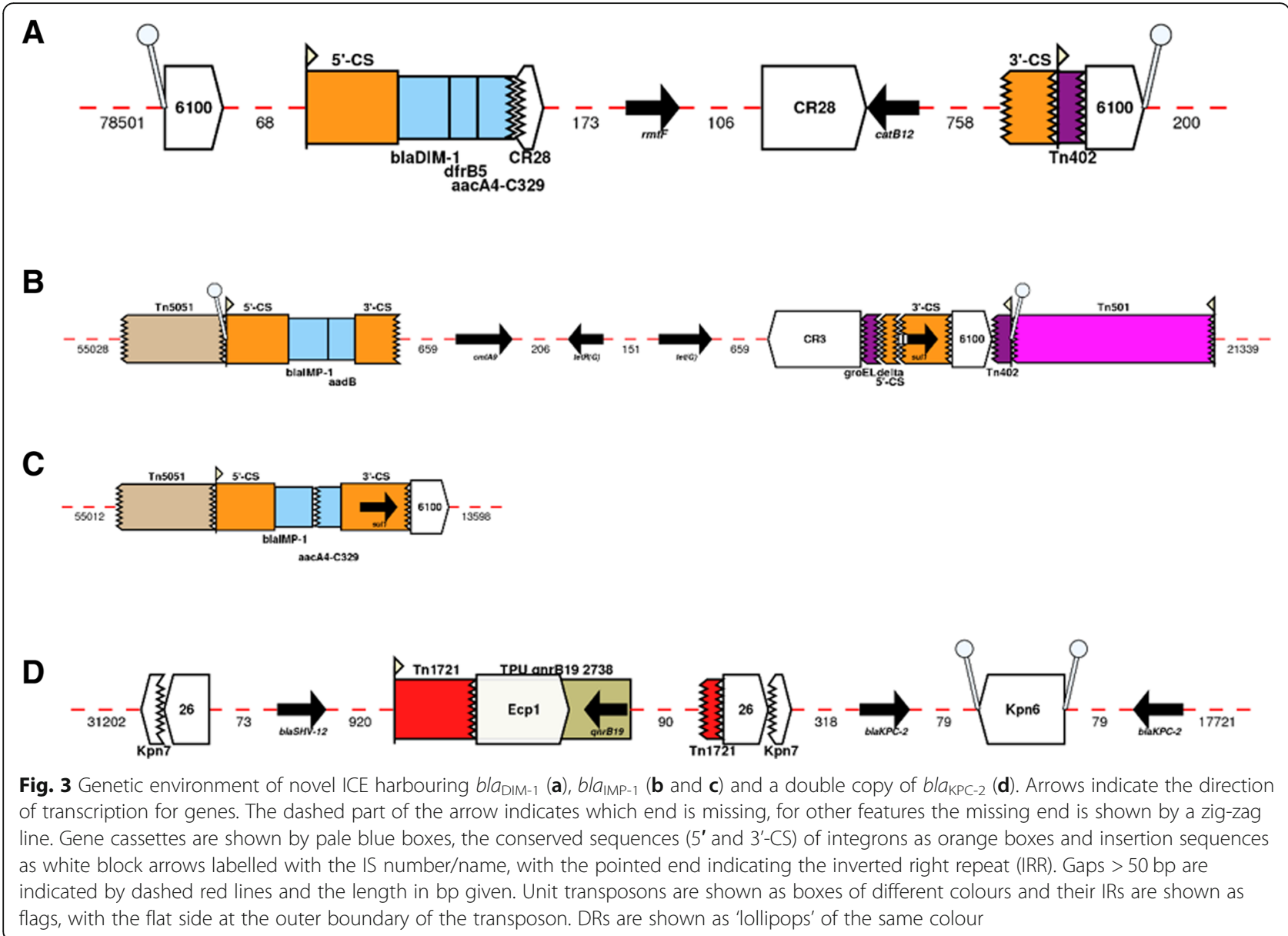

soli species, since the ANIb value was above the 95\% cut-off for species delineation [55]. The PONHI3 strain carried a double copy of $b l a_{\mathrm{KPC}-2}$ within an ICE from $\mathrm{MPF}_{\mathrm{T}}$ class (Fig. 3d). This ICE was integrated between a gene encoding for a biopolymer transport protein ExbD/ TolR (C3F42_RS18665) and a gene encoding for an alpha/ beta hydrolase (C3F42_RS18995).

\section{An atypical $\mathrm{Gl}$ encoding carbapenemases}

Besides ICEs, we also identified an atypical 19.8-kb long GI harbouring bla VIM-2 $_{\text {in } P \text {. aeruginosa AZPAE13853 }}$ and AZPAE13858 strains from India (Additional file 2: Fig. S1). A similar element was also observed in P. aeruginosa BTP038 strain from the USA, with the exception that the Tn402-like transposon harbouring bla $_{\mathrm{VIM}-2}$ was oriented in an inverted position. Five base-pair DRs (5'-CTCTG in AZPAE13853 and AZPAE13858 and 5'-CTGAG in BTP038 strains) were found flanking this transposon structure. Importantly, in these strains the GIs were flanked by identical signal recognition particle RNAs (srpRNAs), indicating a strong site preference for these elements.

\section{Discussion}

Our results show that $b l a_{\mathrm{VIM}}$ and $b l a_{\mathrm{IMP}}$ are widely disseminated, both geographically and phylogenetically (across Pseudomonas spp.). Moreover, and as previously described, $b l a_{\mathrm{VIM}-2}$ was the most frequently reported CEG (Fig. 1 and Additional file 1: Table S1) [4]. On the other hand, bla $a_{\mathrm{SPM}-1}$ is still restricted to $P$. aeruginosa and Brazil (or patients who had been previously hospitalized in Brazil) [56]. Curiously, some strains (highlighted on Fig. 1) belong to a double ST profile (ST235/ST2613), since the strains carry a double copy with different allele sequences of the house-keeping gene $\operatorname{acs} A$, encoding for an acetyl-coenzyme A synthetase. These genes only display $80.3 \%$ nucleotide identity. We plan to conduct comparative genomic studies to explore the idiosyncrasies of these double ST profile strains.

Not all CEGs are likely to be geographically and phylogenetically disseminated, but those that are more promiscuous present a serious threat. The geographical distribution of the high-risk clones and the diversity of CEGs propose that the spread of these STs is global and the acquisition of the resistance genes is mainly 
local [4, 57]. Previous studies suggest that environmental species may have a role as an important reservoir for the dissemination of clinically relevant carbapenemases, which are vertically amplified upon transfer to $P$. aeruginosa high-risk clones [12, 14]. The prevalence of these elements among high-risk clones may be partially explained by the genetic capitalism theory, given that a widely disseminated ST should have a greater probability of acquiring new CEGs and to be further selected and amplified due to the high antibiotic pressure in the hospital environment [58]. Other theories support that the high-risk clones have a naturally increased ability to acquire foreign DNA, since these STs appear to have lost the CRISPR (clustered regularly interspaced short palindromic repeats)-Cas (CRISPR associated proteins) system, which act as an adaptive immune system in prokaryotic cells and protects them from invasion by bacteriophages and plasmids [59-61].

This study underestimates the extent of host range because only ICEs in sequenced genomes were detected. Also, identification of new ICEs could only be achieved in complete genomes or contigs with a sequence length large enough to include the full (or near complete) sequence of the ICE. As so, it is important to highlight the need to perform third generation sequencing on CEG-harbouring genomes to avoid fragmentation of the genetic environment surrounding the gene and to provide a wider view of complete ICEs and other MGEs. All ICE elements here identified fulfilled the criteria to be considered conjugative as proposed by Cury et al: a relaxase, a VirB4/TraU, a T4CP and minimum set of MPF type-specific genes [38]. ICEs tend to integrate within the host's chromosome by the action of a tyrosine recombinase, even though some elements may use serine or DDE recombinases instead [27]. Though rare, some elements encode more than one integrase, most likely resulting from independent integration of different MGEs [38]. Conserved sites are hotspots for ICE integration due to their high conservation among closely related bacteria, and so expanding the host range and be stably maintained after conjugative transfer [62,63]. ICEs were often integrated next to phages highly similar to the Pseudomonas phage Pf1 (NC_001331.1), a class II filamentous bacteriophage belonging to the Inoviridae family [61]. Pf1-like phages are widely disseminated among $P$. aeruginosa strains and may have a role in bacterial evolution and virulence [64-66]. Interestingly, no representative of the pKLC102 family was linked to the dissemination of CEGs. This may be due to a higher affinity of the transposons carrying the CEGs for hotspots located within representatives of the other two families.

MGEs specifically targeting conserved regions of the genome such as tRNAs are common and this specificity represents an evolutionary strategy whereby the target site of an element is almost guaranteed to be present, due to its essentiality, and very unlikely to change due to biochemical constraints of the gene product. We think a similar situation exists for the elements found between the small srpRNAs described on the atypical GI elements here identified and is in contrast to the more permissive nature of target site selection shown for example, by elements of the $\operatorname{Tn} 916 / \operatorname{Tn} 1545$ family [67].

\section{Conclusions}

Here, we revealed that different Tn3-like and composite transposons harbouring a wide array of CEGs were transposed into MPF G and T ICE classes, which were most likely responsible for the dissemination of these genes through HGT and/or clonal expansion of successful Pseudomonas clones. This study sheds light on the underappreciated contribution of ICEs for the spread of CEGs among pseudomonads (and potentially further afield). With the ever-growing number of third-generation sequenced genomes and the development of more sophisticated bioinformatics, the real contribution of these ICEs will likely rapidly emerge.

Recently, it was shown that interfering with the transposase-DNA complex architecture of the conjugative transposon (also know as ICE) Tn1549 leads to transposition inhibition to a new host [68]. In the future, it would be interesting to determine if the same mechanism is observed for tyrosine recombinases present in ICE $c l c$ and Tn4371 derivatives, as well as in other MPF ICE classes, as a potential approach to interfere with the spread of antimicrobial resistance.

\section{Methods}

\section{Carbapenemases database}

Antimicrobial resistance translated sequences were retrieved from the Bacterial Antimicrobial Resistance Reference Gene Database available on NCBI (ftp:// ftp.ncbi.nlm.nih.gov/pathogen/Antimicrobial_resistance/A MRFinder/data/2018-04-16.1/). The resulting 4250 proteins were narrowed down to 695 different carbapenemases to create a binary DIAMOND (v. 0.9.21, https:// github.com/bbuchfink/diamond) database [69]. Only the sequences presenting 'carbapenem-hydrolyzing' or 'metallo-beta-lactamase' on fasta-headers were used to build this local database.

\section{Genome collection and blast search}

A total of 4565 Pseudomonas genomes was downloaded from NCBI (accessed on the 24th of April, 2018). These genomes were blasted against the local carbapenemase 
database using the following command: 'diamond blastx -d DB.dmnd -o hits.txt --id 100 --subject-cover 100 -f 6 --sensitive'.

\section{Bioinformatic prediction of ICE and genetic environment analyses}

The CEG-harbouring Pseudomonas genomes were annotated through Prokka v. 1.12 (https://github.com/tsee mann/prokka) [70]. The translated coding sequences were analysed in TXSScan/CONJscan platform to inspect the presence of ICEs (https://galaxy.pasteur.fr/ root?tool_id=toolshed.pasteur.fr\%2Frepos\%2Fodoppelt\% 2Fconjscan\%2FConjScan\%2F1.0.2) [35]. All ICEs harbouring CEGs predicted by TXSScan/CONJscan were inspected for DRs that define the boundaries of the element. The complete nucleotide sequence in Genbank format of corresponding records was imported into Geneious v. 9.1.8 to help delimiting genomic regions flanking the ICEs [71]. Complete ICE sequences were aligned with EasyFig v. 2.2.2 (http://mjsull.github.io/ Easyfig/files.html) [72]. Screening of complete ICEs for ARG was achieved by ABRicate v. 0.8 (https://github. com/tseemann/abricate). Phage and insertion sequences were inspected through PHASTER (http://phaster.ca/) and ISfinder (https://www-is.biotoul.fr/), respectively [73, 74]. Multiple Antibiotic Resistance Annotator (MARA, http://galileoamr.arcbio.com/mara/) was used to explore the genetic background of the CEGs [75]. Orthologous assignment and functional annotation of integrase sequences was achieved through EggNOG v. 4.5.1 (http://eggnogdb.embl.de/\#/app/home) and InterProScan 5 (https://www.ebi.ac.uk/interpro/search/ sequence-search) [76, 77].

\section{Phylogenomics}

All CEG-harbouring $P$. aeruginosa genomes were mapped against the $P$. aeruginosa PAO1 reference strain (accession number NC_002516.2), to infer a phylogeny based on the concatenated alignment of high quality SNPs using CSI Phylogeny and standard settings [78]. The phylogenetic tree was plotted using the iTOL platform (https://itol.embl.de/).

\section{MLST and taxonomic assignment of unidentified species} To predict the ST of the strains harbouring ICEs, the $P$. aeruginosa MLST website (https://pubmlst.org/paeruginosa/) developed by Keith Jolley and hosted at the University of Oxford was used [79]. Taxonomic assignment of unidentified species carrying ICEs was achieved by JSpeciesWS v. 3.0.17 (http://jspecies.ribohost.com/jspeciesws/) [80].

\section{Additional files}

Additional file 1: Table S1. General features of the hits. Hits associated with ICEs are highlighted in blue. Strains for which more than one CEG was identified are represented in red. (DOCX 52 kb)

Additional file 2: Figure S1. Genetic environment of a novel genomic

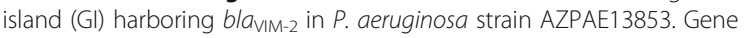
cassettes are shown by pale blue boxes, the conserved sequence (5'-CS) of the integron as orange boxes. Gaps $>50 \mathrm{bp}$ are indicated by dashed red lines and the length in bp given. Transposons IRs are shown as flags, with the flat side at the outer boundary of the transposon. (DOCX $34 \mathrm{~kb}$ )

Additional file 3: Original tree in Newick format. (ZIP 3 kb)

\section{Abbreviations}

ARG: Antibiotic resistance gene; CEG: Carbapenemase-encoding gene; Gl: Genomic island; HGT: Horizontal gene transfer; ICE: Integrative and conjugative element; MGE: Mobile genetic element; MLST: Multilocus sequence typing; MPF: Mating pair formation; ST: Sequence type; T4CP: TypeIV coupling protein; T4SS: Type-IV secretion system

\section{Acknowledgments}

We thank Álvaro San Millan for helpful discussions. We also thank Benjamin Buchfink (DIAMOND), Jean Cury (TXSScan/CONJscan) and Sally Partridge (MARA) for their valuable assistance.

\section{Funding}

This study received financial support from the European Union (FEDER funds POCl/01/0145/FEDER/007728) and National Funds (FCT/MEC, Fundação para a Ciência e Tecnologia and Ministério da Educação e Ciência) under the Partnership Agreement PT2020 UID/MULTI/04378/2013. J. B. and F. G. were supported by grants from Fundação para a Ciência e a Tecnologia (SFRH/ BD/104095/2014 and SFRH/BPD/95556/2013, respectively).

\section{Availability of data and materials}

The accession numbers of all genomic data used and/or analysed during the current study are provided in the manuscript or in the supplementary files.

\section{Authors' contributions}

$J B, A P R, F G$ and LP designed the study; JB and RLS performed the in silico analysis; JB wrote the manuscript. All the authors approved the final manuscript.

Ethics approval and consent to participate

Not applicable.

Consent for publication

Not applicable.

\section{Competing interests}

The authors declare that they have no competing interests.

\section{Publisher's Note}

Springer Nature remains neutral with regard to jurisdictional claims in published maps and institutional affiliations.

\section{Author details}

${ }^{1}$ UCIBIO/REQUIMTE, Laboratório de Microbiologia, Faculdade de Farmácia da Universidade do Porto, Rua Jorge Viterbo Ferreira no 228, 4050-313 Porto, Portugal. ${ }^{2}$ Department of Parasitology, Liverpool School of Tropical Medicine, Liverpool, UK. ${ }^{3}$ Centre for Drugs and Diagnostics, Liverpool School of Tropical Medicine, Liverpool, UK. ${ }^{4}$ Department of Microbiology, University Hospital Ramón y Cajal, Ramón y Cajal Health Research Institute (IRYCIS), Madrid, Spain. ${ }^{5}$ Biomedical Research Networking Center for Epidemiology and Public Health (CIBER-ESP), Madrid, Spain. 


\section{Received: 8 October 2018 Accepted: 29 November 2018} Published online: 19 December 2018

\section{References}

1. Parte AC. LPSN — list of prokaryotic names with standing in nomenclature. Nucleic Acids Res. 2014;42:D613-6.

2. Gomila M, Peña A, Mulet M, Lalucat J, García-Valdés E. Phylogenomics and systematics in Pseudomonas. Front Microbiol. 2015;6:214.

3. Moradali MF, Ghods S, Rehm BHA. Pseudomonas aeruginosa lifestyle: a paradigm for adaptation, survival, and persistence. Front Cell Infect Microbiol. 2017;7:39

4. Oliver A, Mulet X, López-Causapé C, Juan C. The increasing threat of Pseudomonas aeruginosa high-risk clones. Drug Resist Updat. 2015;21-22:41-59.

5. Juan C, Peña C, Oliver A. Host and pathogen biomarkers for severe Pseudomonas aeruginosa infections. J Infect Dis. 2017:215:S44-51.

6. Boucher HW, Talbot GH, Bradley JS, Edwards JE, Gilbert D, Rice LB, et al. Bad bugs, no drugs: no ESKAPE! An update from the Infectious Diseases Society of America. Clin Infect Dis. 2009:48:1-12

7. Breidenstein EBM, de la Fuente-Núñez C, Hancock REW. Pseudomonas aeruginosa: all roads lead to resistance. Trends Microbiol. 2011;19:419-26.

8. Cornaglia G, Giamarellou H, Rossolini GM. Metallo- $\beta$-lactamases: a last frontier for $\beta$-lactams? Lancet Infect Dis. 2011;11:381-93.

9. Kidd TJ, Ritchie SR, Ramsay KA, Grimwood K, Bell SC, Rainey PB. Pseudomonas aeruginosa exhibits frequent recombination, but only a limited association between genotype and ecological setting. PLoS One. 2012;7:e44199.

10. EFSA Panel on Biological Hazards (BIOHAZ). Scientific opinion on Carbapenem resistance in food animal ecosystems. EFSA J. 2013:11(12):3501.

11. World Health Organization. Global priority list of antibiotic-resistant bacteria to guide research, discovery, and development of new antibiotics. http:// www.who.int/news-room/detail/27-02-2017-who-publishes-list-of-bacteriafor-which-new-antibiotics-are-urgently-needed. Accessed 11 Aug 2018.

12. Scotta C, Juan C, Cabot G, Oliver A, Lalucat J, Bennasar A, et al. Environmental microbiota represents a natural reservoir for dissemination of clinically relevant metallo-beta-lactamases. Antimicrob Agents Chemother. 2011:55:5376-9.

13. Poirel L, Rodríguez-Martínez J-M, Al Naiemi N, Debets-Ossenkopp YJ, Nordmann P. Characterization of DIM-1, an integron-encoded metallo-betalactamase from a Pseudomonas stutzeri clinical isolate in the Netherlands. Antimicrob Agents Chemother. 2010;54:2420-4.

14. Juan C, Zamorano L, Mena A, Alberti S, Perez JL, Oliver A. Metallo- $\beta$ lactamase-producing Pseudomonas putida as a reservoir of multidrug resistance elements that can be transferred to successful Pseudomonas aeruginosa clones. J Antimicrob Chemother. 2010;65:474-8.

15. Queenan AM, Bush K. Carbapenemases: the versatile beta-lactamases. Clin Microbiol Rev. 2007;20:440-58.

16. Mazel D. Integrons: agents of bacterial evolution. Nat Rev Microbiol. 2006;4: 608-20.

17. Stokes HW, Hall RM. A novel family of potentially mobile DNA elements encoding site-specific gene-integration functions: integrons. Mol Microbiol. 1989;3:1669-83.

18. Escudero JA, Loot C, Nivina A, Mazel D. The Integron: adaptation on demand. Microbiol Spectr. 2015;3:MDNA3-0019-2014.

19. Kung VL, Ozer EA, Hauser AR. The accessory genome of Pseudomonas aeruginosa. Microbiol Mol Biol Rev. 2010;74:621-41.

20. Partridge SR, Kwong SM, Firth N, Jensen SO. Mobile genetic elements associated with antimicrobial resistance. Clin Microbiol Rev. 2018;31:e00088-17.

21. Toleman MA, Walsh TR. Combinatorial events of insertion sequences and ICE in gram-negative bacteria. FEMS Microbiol Rev. 2011;35:912-35.

22. Enault F, Briet A, Bouteille $L$, Roux S, Sullivan MB, Petit M-A. Phages rarely encode antibiotic resistance genes: a cautionary tale for virome analyses. ISME J. 2017:11:237-47.

23. San Millan A. Evolution of plasmid-mediated antibiotic resistance in the clinical context. Trends Microbiol. 2018;26:978-85. In Press.

24. Bellanger X, Payot S, Leblond-Bourget N, Guédon G. Conjugative and mobilizable genomic islands in bacteria: evolution and diversity. FEMS Microbiol Rev. 2014:38:720-60.

25. Langille MGl, Hsiao WWL, Brinkman FSL. Detecting genomic islands using bioinformatics approaches. Nat Rev Microbiol. 2010;8:373-82

26. Wozniak RAF, Waldor MK. Integrative and conjugative elements: mosaic mobile genetic elements enabling dynamic lateral gene flow. Nat Rev Microbiol. 2010;8:552-63.
27. Johnson CM, Grossman AD. Integrative and conjugative elements (ICEs): what they do and how they work. Annu Rev Genet. 2015;49:577-601.

28. Delavat F, Miyazaki R, Carraro N, Pradervand N, van der Meer JR. The hidden life of integrative and conjugative elements. FEMS Microbiol Rev. 2017;41:512-37.

29. Brophy JAN, Triassi AJ, Adams BL, Renberg RL, Stratis-Cullum DN, Grossman AD, et al. Engineered integrative and conjugative elements for efficient and inducible DNA transfer to undomesticated bacteria. Nat Microbiol. 2018;3:1043-53.

30. Guédon G, Libante V, Coluzzi C, Payot S, Leblond-Bourget N. The obscure world of integrative and Mobilizable elements, highly widespread elements that pirate bacterial conjugative systems. Genes (Basel). 2017;8:337.

31. Delavat F, Mitri S, Pelet S, van der Meer JR. Highly variable individual donor cell fates characterize robust horizontal gene transfer of an integrative and conjugative element. Proc Natl Acad Sci U S A. 2016;113:E3375-83.

32. Burrus V. Mechanisms of stabilization of integrative and conjugative elements. Curr Opin Microbiol. 2017:38:44-50.

33. Smillie C, Garcillán-Barcia MP, Francia MV, Rocha EPC, de la Cruz F. Mobility of plasmids. Microbiol Mol Biol Rev. 2010;74:434-52.

34. Garcillán-Barcia MP, Alvarado A, de la Cruz F. Identification of bacteria plasmids based on mobility and plasmid population biology. FEMS Microbiol Rev. 2011;35:936-56.

35. Guglielmini J, Néron B, Abby SS, Garcillán-Barcia MP, de la Cruz F, Rocha EPC. Key components of the eight classes of type IV secretion systems involved in bacterial conjugation or protein secretion. Nucleic Acids Res. 2014:42:5715-27.

36. Guglielmini J, Quintais L, Garcillán-Barcia MP, de la Cruz F, Rocha EPC. The repertoire of ICE in prokaryotes underscores the Unity, diversity, and ubiquity of conjugation. PLoS Genet. 2011;7:e1002222.

37. Carraro N, Poulin D, Burrus V. Replication and active partition of integrative and conjugative elements (ICES) of the SXT/R391 family: the line between ICEs and conjugative plasmids is getting thinner. PLoS Genet. 2015;11:e1005298.

38. Cury J, Touchon M, Rocha EPC. Integrative and conjugative elements and their hosts: composition, distribution and organization. Nucleic Acids Res. 2017;45:8943-56.

39. Baltrus DA. Exploring the costs of horizontal gene transfer. Trends Ecol Evol. 2013;28:489-95.

40. Oliveira PH, Touchon M, Cury J, Rocha EPC. The chromosomal organization of horizontal gene transfer in bacteria. Nat Commun. 2017;8:841.

41. Harrison EM, Carter MEK, Luck S, Ou H-Y, He X, Deng Z, et al. Pathogenicity islands PAPI-1 and PAPI-2 contribute individually and synergistically to the virulence of Pseudomonas aeruginosa strain PA14. Infect Immun. 2010;78:1437-46.

42. He J, Baldini RL, Déziel E, Saucier M, Zhang Q, Liberati NT, et al. The broad host range pathogen Pseudomonas aeruginosa strain PA14 carries two pathogenicity islands harboring plant and animal virulence genes. Proc Natl Acad Sci U S A. 2004;101:2530-5.

43. Botelho J, Grosso F, Peixe L. Characterization of the pJB12 plasmid from Pseudomonas aeruginosa reveals Tn6352, a novel putative transposon associated with mobilization of the bla $\mathrm{VIM}_{\mathrm{M}-2}$-harboring In58 Integron. Antimicrob Agents Chemother. 2017;61:e02532-16.

44. Botelho J, Grosso F, Quinteira S, Mabrouk A, Peixe L. The complete nucleotide sequence of an IncP-2 megaplasmid unveils a mosaic

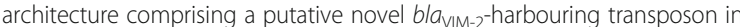
Pseudomonas aeruginosa. J Antimicrob Chemother. 2017;72:2225-9.

45. San Millan A, Toll-Riera M, Escudero JA, Cantón R, Coque TM, MacLean RC. Sequencing of plasmids PAMBL1 and PAMBL2 from Pseudomonas aeruginosa reveals a bla $a_{\mathrm{VIM}-1}$ amplification causing high-level carbapenem resistance. J Antimicrob Chemother. 2015;70:3000-3.

46. Sun F, Zhou D, Wang Q, Feng J, Feng W, Luo W, et al. Genetic

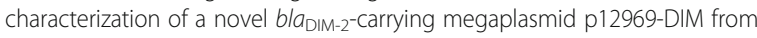
clinical Pseudomonas putida. J Antimicrob Chemother. 2016;71:909-12.

47. Roy Chowdhury P, Scott M, Worden P, Huntington P, Hudson B, Karagiannis $T$, et al. Genomic islands 1 and 2 play key roles in the evolution of extensively drug-resistant ST235 isolates of Pseudomonas aeruginosa. Open Biol. 2016;6:150175.

48. Botelho J, Grosso F, Peixe L. Unravelling the genome of a Pseudomonas aeruginosa isolate belonging to the high-risk clone ST235 reveals an integrative conjugative element housing a bla $a_{\mathrm{GES}-6}$ carbapenemase. J Antimicrob Chemother. 2018;73:77-83.

49. Botelho J, Grosso F, Quinteira S, Brilhante M, Ramos H, Peixe L. Two decades of bla $\mathrm{VIM}_{\mathrm{IM}-2}$-producing Pseudomonas aeruginosa dissemination: an interplay between mobile genetic elements and successful clones. J Antimicrob Chemother. 2018;73:873-82. 
50. Ding Y, Teo JWP, Drautz-Moses DI, Schuster SC, Givskov M, Yang L. Acquisition of resistance to carbapenem and macrolide-mediated quorum sensing inhibition by Pseudomonas aeruginosa via ICETn43716385. Commun Biol. 2018;1:57.

51. Fonseca EL, Marin MA, Encinas F, Vicente ACP. Full characterization of the integrative and conjugative element carrying the metallo- $\beta$-lactamase bla $a_{\text {PM-1 }}$ and bicyclomycin bcrl resistance genes found in the pandemic Pseudomonas aeruginosa clone SP/ST277. J Antimicrob Chemother. 2015;70: 2547-50.

52. Toleman MA, Bennett PM, Walsh TR. ISCR elements: novel gene-capturing systems of the 21st century? Microbiol Mol Biol Rev. 2006;70:296-316.

53. Partridge SR. Analysis of antibiotic resistance regions in gram-negative bacteria. FEMS Microbiol Rev. 2011;35:820-55.

54. Shimizu W, Kayama S, Kouda S, Ogura Y, Kobayashi K, Shigemoto N, et al. Persistence and epidemic propagation of a Pseudomonas aeruginosa sequence type 235 clone harboring an IS26 composite transposon carrying the bla $a_{\mid M P-1}$ integron in Hiroshima, Japan, 2005 to 2012. Antimicrob Agents Chemother. 2015;59:2678-87.

55. Varghese NJ, Mukherjee S, Ivanova N, Konstantinidis KT, Mavrommatis K, Kyrpides NC, et al. Microbial species delineation using whole genome sequences. Nucleic Acids Res. 2015;43:6761-71.

56. Nascimento APB, Ortiz MF, Martins WMBS, Morais GL, Fehlberg LCC, Almeida LGP, et al. Intraclonal genome stability of the Metallo- $\beta$-lactamase SPM-1-producing Pseudomonas aeruginosa ST277, an endemic clone disseminated in Brazilian hospitals. Front Microbiol. 2016;7:1946.

57. Turton JF, Wright L, Underwood A, Witney AA, Chan Y-T, Al-Shahib A, et al. High-resolution analysis by whole-genome sequencing of an international lineage (sequence type 111) of Pseudomonas aeruginosa associated with Metallo-Carbapenemases in the United Kingdom. J Clin Microbiol. 2015;53: 2622-31.

58. Baquero F. From pieces to patterns: evolutionary engineering in bacterial pathogens. Nat Rev Microbiol. 2004;2:510-8.

59. Miyoshi-Akiyama T, Tada T, Ohmagari N, Viet Hung N, Tharavichitkul P, Pokhrel BM, et al. Emergence and spread of epidemic multidrug-resistant Pseudomonas aeruginosa. Genome Biol Evol. 2017;9:3238-45.

60. Bondy-Denomy J, Davidson AR. To acquire or resist: the complex biological effects of CRISPR-Cas systems. Trends Microbiol. 2014;22:218-25.

61. van Belkum A, Soriaga LB, LaFave MC, Akella S, Veyrieras J-B, Barbu EM, et al. Phylogenetic distribution of CRISPR-Cas systems in antibiotic-resistant Pseudomonas aeruginosa. MBio. 2015;6:e01796-15.

62. Touchon M, Hoede C, Tenaillon O, Barbe V, Baeriswyl S, Bidet P, et al. Organised genome dynamics in the Escherichia coli species results in highly diverse adaptive paths. PLoS Genet. 2009:5:e1000344.

63. Rocha EPC. The replication-related organization of bacterial genomes. Microbiology. 2004;150:1609-27.

64. Knezevic P, Voet M, Lavigne R. Prevalence of Pf1-like (pro)phage genetic elements among Pseudomonas aeruginosa isolates. Virology. 2015;483:64-71.

65. Whiteley M, Bangera MG, Bumgarner RE, Parsek MR, Teitzel GM, Lory S, et al. Gene expression in Pseudomonas aeruginosa biofilms. Nature. 2001;413:860-4.

66. Secor PR, Michaels LA, Smigiel KS, Rohani MG, Jennings LK, Hisert KB, et al. Filamentous bacteriophage produced by Pseudomonas aeruginosa alters the inflammatory response and promotes noninvasive infection in vivo. Infect Immun. 2017:85:e00648-16.

67. Roberts AP, Mullany P. Tn916-like genetic elements: a diverse group of modular mobile elements conferring antibiotic resistance. FEMS Microbiol Rev. 2011;35:856-71.

68. Rubio-Cosials A, Schulz EC, Lambertsen L, Smyshlyaev G, Rojas-Cordova C, Forslund $\mathrm{K}$, et al. Transposase-DNA complex structures reveal mechanisms for conjugative transposition of antibiotic resistance. Cell. 2018;173:208-220.e20.

69. Buchfink B, Xie C, Huson DH. Fast and sensitive protein alignment using DIAMOND. Nat Methods. 2015;12:59-60.

70. Seemann T. Prokka: rapid prokaryotic genome annotation. Bioinformatics. 2014;30:2068-9.

71. Kearse M, Moir R, Wilson A, Stones-Havas S, Cheung M, Sturrock S, et al. Geneious basic: an integrated and extendable desktop software platform for the organization and analysis of sequence data. Bioinformatics. 2012;28: 1647-9.

72. Sullivan MJ, Petty NK, Beatson SA. Easyfig: a genome comparison visualizer. Bioinformatics. 2011;27:1009-10.

73. Arndt D, Grant JR, Marcu A, Sajed T, Pon A, Liang Y, et al. PHASTER: a better, faster version of the PHAST phage search tool. Nucleic Acids Res. 2016;44: W16-21.
74. Siguier P, Perochon J, Lestrade L, Mahillon J, Chandler M. ISfinder: the reference Centre for bacterial insertion sequences. Nucleic Acids Res. 2006; 34:D32-6.

75. Partridge SR, Tsafnat G. Automated annotation of mobile antibiotic resistance in gram-negative bacteria: the multiple antibiotic resistance annotator (MARA) and database. J Antimicrob Chemother. 2018;73:883-90.

76. Huerta-Cepas J, Forslund K, Coelho LP, Szklarczyk D, Jensen LJ, von Mering $C$, et al. Fast genome-wide functional annotation through Orthology assignment by eggNOG-mapper. Mol Biol Evol. 2017;34:2115-22.

77. Jones P, Binns D, Chang H-Y, Fraser M, Li W, McAnulla C, et al. InterProScan 5: genome-scale protein function classification. Bioinformatics. 2014;30: 1236-40.

78. Kaas RS, Leekitcharoenphon P, Aarestrup FM, Lund O. Solving the problem of comparing whole bacterial genomes across different sequencing platforms. PLoS One. 2014;9:e104984.

79. Jolley KA, Maiden MCJ. BIGSdb: scalable analysis of bacterial genome variation at the population level. BMC Bioinformatics. 2010;11:595.

80. Richter M, Rosselló-Móra R, Oliver Glöckner F, Peplies J. JSpeciesWS: a web server for prokaryotic species circumscription based on pairwise genome comparison. Bioinformatics. 2016;32:929-31.

81. Weingarten RA, Johnson RC, Conlan S, Ramsburg AM, Dekker JP, Lau AF, et al. Genomic analysis of hospital plumbing reveals diverse reservoir of bacterial plasmids conferring Carbapenem resistance. MBio. 2018;9:e02011-7.

82. Giani T, Arena F, Pollini S, Di Pilato V, D'Andrea MM, Henrici De Angelis L, et al. Italian nationwide survey on Pseudomonas aeruginosa from invasive infections: activity of ceftolozane/tazobactam and comparators, and molecular epidemiology of carbapenemase producers. J Antimicrob Chemother. 2018;73:664-71.

\section{Ready to submit your research? Choose BMC and benefit from:}

- fast, convenient online submission

- thorough peer review by experienced researchers in your field

- rapid publication on acceptance

- support for research data, including large and complex data types

- gold Open Access which fosters wider collaboration and increased citations

- maximum visibility for your research: over $100 \mathrm{M}$ website views per year

At BMC, research is always in progress.

Learn more biomedcentral.com/submissions 